${ }^{3}$ Bangabandhu Sheikh Mujib Medical University, Dhaka, Bangladesh. ${ }^{4}$ Damascus University, Damascus, Syria. ${ }^{5}$ Kenya Paediatrics Association, Nairobi, Kenya. ${ }^{6}$ State Department for Public Health of Baden-Wurttemberg, Baden-Wurttemberg, Germany.

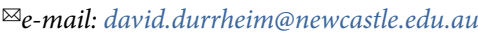

Published online: 15 February 2021

https://doi.org/10.1038/s41591-021-01237-5

References

1. Snape, M. D. \& Viner, R. M. Science 370, 286-288 (2020).

2. World Health Organization. Wkly. Epidemiol. Rec. 95 , $585-608(2020)$.
3. Patel, M. K. et al. MMWR Morb. Mortal. Wkly. Rep. 69 1700-1705 (2020)

4. Durrheim, D. N. Lancet Infect. Dis. 20, e138-e141 (2020).

5. Mina, M. J. et al. Science 366, 599-606 (2019).

6. Mulholland, K., Kretsinger, K., Wondwossen, L. \& Crowcroft, N. Lancet 396, 1782-1784 (2020).

7. World Health Organization. Wkly. Epidemiol. Rec. 92, 649-658 (27 October 2017); https://apps.who.int/iris/bitstream/ handle/10665/259369/WER9243.pdf

8. World Health Organization. Wkly. Epidemiol. Rec. 93, 649-658 (30 November 2018); https://apps.who.int/iris/bitstream/ handle/10665/276217/WER9348.pdf

9. World Health Organization. Wkly. Epidemiol. Rec. 94, 581-590 (6 December 2019); https://extranet.who.int/iris/restricted/ bitstream/handle/10665/330042/WER9449-eng-fre.pdf

10. World Health Organization. Wkly. Epidemiol. Rec. 95, 564-572 (13 November 2020); https://apps.who.int/iris/bitstream/ handle/10665/336590/WER9546-eng-fre.pdf
Acknowledgements

D.N.D. is the chairperson of the Western Pacific Regional Measles and Rubella Elimination Verification Commission. J.K.A. is the chairperson of the Regional Measles and Rubella Elimination Verification Commission of the Americas. S.T. is the chairperson of the South-East Asian Regional Measles and Rubella Elimination Verification Commission. H.B. is the chairperson of the Eastern Mediterranean Regional Measles and Rubella Elimination Verification Commission. D.G. is the chairperson of the African Regional Measles and Rubella Elimination Verification Commission. G.P. is the chairperson of the European Regional Measles and Rubella Elimination Verification Commission.

The authors declare no competing interests.

\title{
Time to evaluate COVID-19 contact-tracing apps
}

To the Editor-Digital contact tracing is a public-health intervention. Real-time monitoring and evaluation of the effectiveness of app-based contact tracing is key for improvement and public trust.

SARS-CoV-2 is likely to become endemic in many parts of the world, and there is still no certainty about how quickly vaccination will become available or how long its protection will last. For the foreseeable future, most countries will rely on a combination of various measures, including vaccination, social distancing, mask wearing and contact tracing.

Digital contact tracing via smartphone apps was established as a new public-health intervention in many countries in 2020. Most of these apps are now at a stage at which they need to be evaluated as public-health tools. We present here five key epidemiological and public-health requirements for COVID-19 contact-tracing apps and their evaluation.

1. Integration with local health policy. App notifications should be consistent with local health policies. The app should be integrated into access to testing, medical care and advice on isolation, and should work in conjunction with conventional contact tracing where available ${ }^{1}$. Apps should be interoperable across countries, as envisaged by the European Commission's eHealth Network.

\section{High user uptake and adherence. Contact-tracing apps can reduce transmission at low levels of uptake, including for those without smartphones ${ }^{2}$. However, large numbers of users increase effectiveness $^{3,4}$. An effective communication strategy that explains the apps' role and}

addresses privacy concerns is essential for increasing adoption ${ }^{5}$. Design, implementation and deployment should make the apps accessible to harder-to-reach communities. Adherence to quarantine should be encouraged and supported.

\section{Quarantine infectious people as} accurately as possible. The purpose of contact tracing is to quarantine as many potentially infectious people as possible, but to minimize the time spent in quarantine by uninfected people. To achieve optimal performance, apps' algorithms must be 'tunable', to adjust to the epidemic as it evolves ${ }^{6}$.

4. Rapid notification. The time between the onset of symptoms in an index case and the quarantine of their contacts is of key importance in COVID-19 contact tracing ${ }^{7,8}$. Where a design feature introduces a delay, it needs to be outweighed by gains in, for example, specificity, uptake or adherence. If the delays exceed the period during which most contacts transmit the disease, the app will fail to reduce transmission.

\section{Ability to evaluate effectiveness transparently. The public must be provided with evidence that notifications are based on the best available data. The tracing algorithm should therefore be transparent, auditable, under oversight and subject to review. Aggregated data (not linked to individual people) are essential for evaluation of and improvement in the performance of the app. Data on local uptake at a sufficiently coarse-grained spatial resolution are equally key. As apps in Europe do not 'geolocate' people, this}

additional information can be provided by the user or through surveys. Real-time monitoring should be performed whenever possible.

A proof-of-principle evaluation is available for the Swiss app ${ }^{9}$. More detailed analysis on the epidemiological effectiveness of contact-tracing apps is needed. For example, index cases seeking healthcare could be asked if they routinely use the app; if so, interviews with contacts identified by traditional tracing would allow assessment of the secondary attack rate among people who were notified. Surveys, epidemiological analyses ${ }^{10}$ and experimental studies such as the Radar COVID pilot in Spain can offer further evaluation.

Digital contact tracing is a sustainable measure that can reduce levels of COVID-19 transmission. A rigorous assessment of its effectiveness allows public-health benefits to be weighed against unwanted effects for individual people and society. Stringent evaluation is needed to develop contact-tracing apps into an accepted and ethical tool for future outbreaks of other infectious diseases.

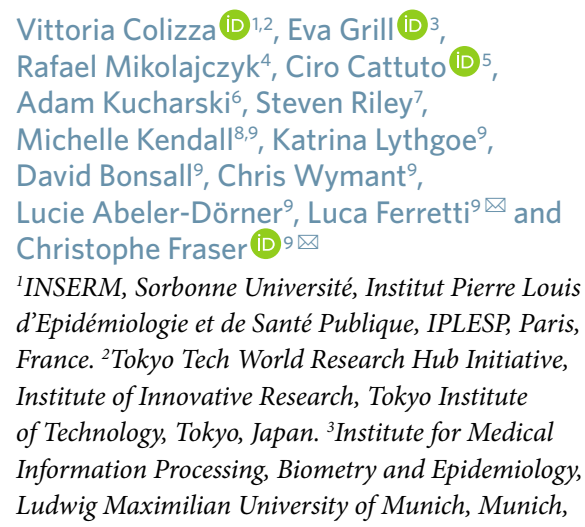


Germany. ${ }^{4}$ Institute for Medical Epidemiology, Biometrics and Informatics, Interdisciplinary Center for Health Sciences, Martin Luther University Halle-Wittenberg, Halle, Germany. ${ }^{5}$ University of Turin and ISI Foundation, Turin, Italy. ${ }^{6}$ Centre for Mathematical Modelling of Infectious Diseases, London School of Hygiene \& Tropical Medicine, London, UK. ${ }^{7} M R C$ Centre for Global Infectious Disease Analysis, School of Public Health, Imperial College London, London, UK. ${ }^{8}$ Department of Statistics, University of Warwick, Warwick, UK. ${ }^{9}$ Big Data Institute, Li Ka Shing Centre for Health
Information and Discovery, Nuffield Department of Medicine, University of Oxford, Oxford, UK.

$凶_{e-\text { mail: luca.ferretti@bdi.ox.ac.uk; }}$

christophe.fraser@bdi.ox.ac.uk

Published online: 15 February 2021

https://doi.org/10.1038/s41591-021-01236-6

References

1. Kucharski, A. J. et al. Lancet Infect. Dis. 20, 1151-1160 (2020).

2. Abueg, $\mathrm{M}$ et al. Preprint at https://doi. org/10.1101/2020.08.29.20184135 (2020).

3. Cencetti, G. et al. Preprint at https://www.medrxiv.org/content/ $10.1101 / 2020.05 .29 .20115915 \mathrm{v} 2(2020)$.
4. Moreno López, J.A. et al. Preprint at https://www.medrxiv.org/ content/10.1101/2020.07.22.20158352v1 (2020).

5. Montagni, i., Roussel, N., Thiébaut, R. \& Tzourio, C. Preprint at https://www.medrxiv.org/content/10.1101/2020.10.23.2021821 $4 \mathrm{v} 2(2020)$

6. Wilson, A.M. et al. Preprint at https://doi org $/ 10.1101 / 2020.07 .17 .20156539(2020)$

7. Ferretti, L. et al. Science 368, eabb6936 (2020).

8. Kretzschmar, M. E. et al. Lancet Public Health 5, e452-e459 (2020).

9. Salathé, M. et al. Swiss Med. Wkly. 150, w20457 (2020). 10. Kendall, M. et al. Lancet Digit. Health 2, e658-e666 (2020).

Competing interests

The authors declare no competing interests. 